\title{
STEAM Pulse Sequence Mixing Time
}

National Cancer Institute

\section{Source}

National Cancer Institute. STEAM Pulse Sequence Mixing Time. NCI Thesaurus. Code C156501.

The time elapsed between the second and third pulses of the stimulated echo acquisition mode (STEAM) pulse sequence. 